\title{
Tolvaptan in ADPKD-TEMPO 3:4 trial results
}

$\mathrm{R}$ esults of the TEMPO 3:4 study, reported at ASN Kidney Week in San Diego, USA in November and published in the New England Journal of Medicine, have shown that tolvaptan may be useful in reducing increases in total kidney volume and slowing renal function decline in autosomal dominant polycystic kidney disease (ADPKD), but is associated with adverse events. "Whether tolvaptan is a helpful long-term treatment in patients with ADPKD will depend on a balance between benefits and risks," state the authors.

"The TEMPO 3:4 trial was initiated based on previous basic and translational research," says corresponding author Vicente Torres. "Studies of animal models showed the importance of cyclic adenosine monophosphate in promoting fluid secretion, cell proliferation and cyst growth in polycystic kidneys. And in 1999, Vince Gattone published an article in Developmental Genetics reporting that a drug that blocks the action of the hormone vasopressin on the vasopressin V2 receptors in the kidney blunted the development of the renal cystic disease in cpk mice. This observation was then confirmed in four rodent models with mutations in the same genes that are associated with human ADPKD ( $P K D 1$ and $P K D 2$ ), autosomal recessive polycystic kidney disease (PKHD1) and nephronophthisis (NPHP3). These preclinical studies provided the basis for phase II trials and then the phase III TEMPO 3:4 trial, which started enrolling patients in January 2007 and was completed in April 2012."

In the TEMPO 3:4 trial, Torres et al. randomly assigned 1,445 patients (aged $18-50$ years) with ADPKD, a total kidney volume of $\geq 750 \mathrm{ml}$ and an estimated creatinine clearance of $\geq 60 \mathrm{ml} / \mathrm{min}$, to treatment with the V2-receptor antagonist tolvaptan (at the highest of three twicedaily doses that the patient was able to tolerate) or placebo. Overall, 961 patients were assigned to tolvaptan and 484

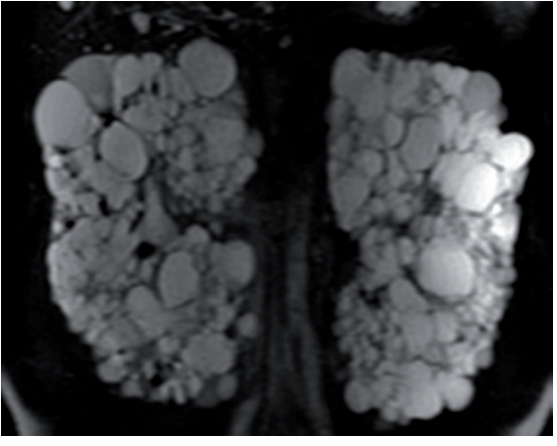

Advanced autosomal dominant polycystic kidney disease. Image courtesy of V. E. Torres, Division of Nephrology and Hypertension, Mayo Clinic, Rochester, MN, USA.

patients were assigned to placebo. The primary efficacy end point was the annual rate of change in total kidney volume, a measurement of kidney cyst growth.

Over 3 years of follow-up, total kidney volume increased more in the placebo group than in the tolvaptan group $(5.5 \%$ per year versus $2.8 \%$ per year; $P<0.001)$.

Tolvaptan use was also associated with a reduction in the risk of a composite secondary end point of time to events of ADPKD progression (worsening kidney function, clinically significant kidney pain, worsening hypertension, and worsening albuminuria), with 44 such events per 100 person-years of follow-up occurring in the tolvaptan group versus 50 events per 100 person-years of follow-up in the placebo group $(P=0.01)$. "The effect on this end point was driven by a $61 \%$ reduction in the risk of an event of worsening kidney function, and a $36 \%$ reduction in the risk of an event of worsening kidney pain," notes Torres.

In addition, tolvaptan reduced the slope of decline in kidney function (measured by the reciprocal of the serum creatinine level) by about $30 \%$ compared with the slope of decline in placebo-treated patients $\left(2.61[\mathrm{mg} / \mathrm{ml}]^{-1}\right.$ decrease per year versus decrease of $3.81[\mathrm{mg} / \mathrm{ml}]^{-1}$ decrease per year; $P<0.001)$.

In total, $1,157(80.1 \%)$ patients completed the 3-year trial, with a greater percentage of the placebo group completing the trial compared with the tolvaptan group (86.2\% versus $77.0 \%$, respectively). Although rates of adverse events were similar in the two groups, discontinuation of treatment owing to adverse events was more common in tolvaptan-treated patients than in placebo-treated patients ( $15.4 \%$ versus $5.0 \%$, respectively). Many of the most common adverse events occurring more often with tolvaptan treatment were related to its aquaretic mode of action: thirst, polyuria, nocturia, urinary frequency, and polydipsia. Tolvaptantreated patients were also more likely to develop the laboratory abnormalities of increased levels of serum sodium, serum uric acid, alanine aminotransferase and aspartate aminotransferase. In the placebo group, the most common adverse events were related to ADPKD and included renal pain, haematuria and urinary tract infection.

"Although tolvaptan is already approved for the treatment of other conditions, it is not approved for the treatment of ADPKD," concludes Torres. “The doses of tolvaptan used in the TEMPO trial were higher than used in previous studies of other diseases. In addition, ADPKD patients are a unique patient population. Further analysis of the benefits and risks of this potential therapy will need to be performed by the sponsor and regulatory agencies. Therefore, although the results are encouraging, at the present time, patients with ADPKD should not be treated with tolvaptan outside of approved research studies. Research studies on how to optimize the administration of tolvaptan and to further assess efficacy and safety are ongoing."

\section{Rebecca Kelsey}

Original article Torres, V. E. et al. Tolvaptan in patients with autosomal dominant polycystic kidney disease. N. Engl. J. Med. doi:10.1056/NEJMoa1205511

Further reading Gattone, V. H. Developmental expression of urine concentration-associated genes and their altered expression in murine infantile-type polycystic kidney disease. Dev. Genet. 24, 309-318 (1999) 